\title{
CONTINUOUS FLOW SWITCHING BY PNEUMATIC ACTUATION OF THE AIR LUBRICATION LAYER ON SUPERHYDROPHOBIC MICROCHANNEL WALLS

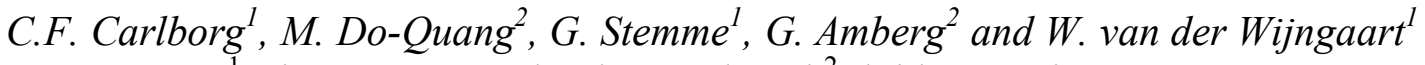 \\ ${ }^{1}$ Microsystem Technology Lab and ${ }^{2}$ Fluid Mecanics Dep., KTH - Royal Institute of Technology, Stockholm, Sweden
}

\begin{abstract}
This paper introduces and experimentally verifies a method for robust, active control of friction reduction in microchannels, enabling new flow control applications and overcoming previous limitations with regard to sustainable liquid pressure. The air pockets trapped at a superhydrophobic micrograting during liquid priming are coupled to an actively controlled pressure source, allowing the pressure difference over the air/liquid interface to be dynamically adjusted. This allows for manipulating the friction reduction properties of the surface, enabling active control of liquid mass flow through the channel. It also permits for sustainable air lubrication at theoretically unlimited liquid pressures, without loss of superhydrophobic properties. With the non-optimized grating used in the experiment, a difference in liquid mass flow of $4.8 \%$ is obtained by alternatively collapsing and recreating the air pockets using the coupled pressure source, which is in line with a FE analysis of the same geometry. A FE analysis of a more optimized geometry predicts a mass flow change of over $30 \%$, which would make possible new microfluidic devices based on local friction control. It is also experimentally shown that our method allows for sustainable liquid pressure 3 times higher than the Laplace pressure of a passive device.
\end{abstract}

\section{INTRODUCTION}

When downscaling microfluidic components the mass transport becomes strongly limited by frictional losses because the surface to volume increases and the near zero slip of liquid is imposed at a solid surface. It has been shown both theoretically $[1,2]$ and experimentally [3-6] that surfaces can be engineered to have a significant slip length, reducing the frictional losses by up to $40 \%$ [4]. Much attention [4,5] has been focused on static laminar drag reduction in continuous flow at superhydrophobic surfaces by passively trapping air pockets at the surface during channel priming, forming a shear-free "lubricating" air boundary to the liquid. However, this passive approach is limited to either low liquid pressures or very small channels. This is because at high liquid pressures $(>10 \mathrm{~s} \mathrm{kPa})$, the air pocket size $a$ (Figure 1) needs to be in the sub-micron range to generate the required Laplace pressure to avoid collapse of the lubricating liquid/air interface [6]:

$$
a<\frac{2 \gamma \cos (\theta)}{P_{\mathrm{lg}}},
$$
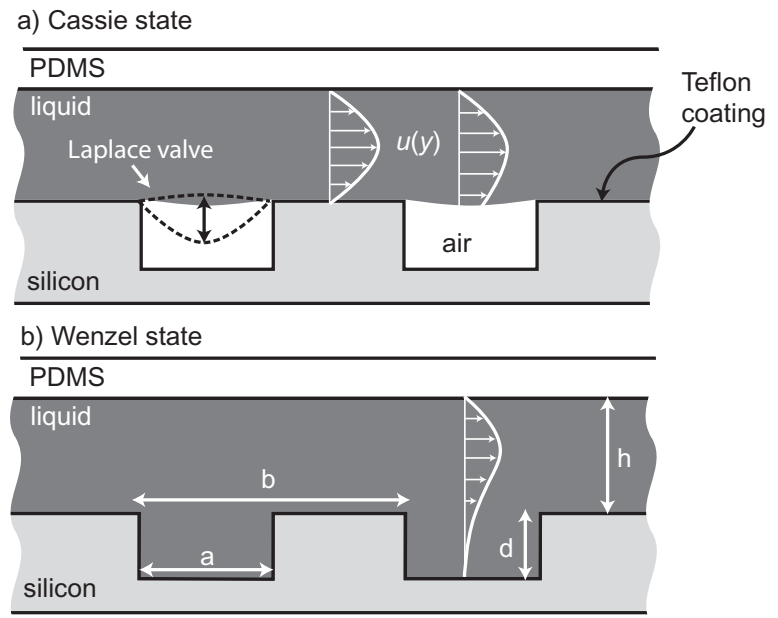

Figure 1: Switching principle on superhydrophobic surface. (a): water-air pressure is less than maximum sustainable Laplace pressure and the air trapped in the grating reduces the effective drag. (b): when reducing the pressure in the air pockets the interface collapses. The increased fluid viscosity in the grating increases the overall drag.

where $P_{l g}$ is the pressure difference over liquid/air interface at the air pocket, $\gamma$ is the surface tension of the liquid and $\theta$ is the liquid/solid contact angle.

On the other hand, it has been shown that for substantial drag reduction, the air pocket size $a$ must be in the same order of magnitude as the channel depth $h$ to achieve substantial drag reduction [1]. The latter can be understood because the liquid at the shear-free liquid-air interface must have the possibility to accelerate from zero (no-slip at the solid contact point) to a velocity that is a substantial portion of the mean flow velocity.

Being able to design a surface that can withstand high liquid pressures but still give substantial drag reduction would have many benefits. In addition to global static drag reduction, extending this concept from active temporal to active spatial dynamic control of the frictional losses would enable microscale applications including integrated valving, control of sample transport and reagent mixing and macroscale applications including reduction of turbulent losses through active dynamically controlled local perturbations at the surface in e.g. pipe flow, lubrication, energy conversion processes propulsion, ship hydrodynamics, etc. [4].

\section{OPERATIONAL PRINCIPLE}

Here we introduce the connection of all air pockets in a microscale superhydrophobic grating to two pneumatic control ports; one upstream and one downstream (Figure 2). This 
allows for three benefits compared to previous passive systems. Firstly, it enables temporal and spatial manipulation of the channel frictional losses on a superhydrophobic surface. Secondly, it permits high liquid pressure on a lubricating air surface without collapse to the Wenzel state. Finally, it increases the robustness of a superhydrophobic surface by enabling active regeneration of the air pockets in case of collapse.

When liquid is introduced in the channel, an air layer is trapped in the superhydrophobic grating (Cassie state, "dry"). The air pressure in the trapped air pocket can now be manipulated using the coupled pressure ports. If the interfacial pressure exceeds the critical Laplace pressure for the air pockets it will move the air/liquid interface down and fill the hydrophobic grooves with water (switch to Wenzel state, "wet"). Conversely, the coupled pressure port can be used to increase the pressure in the air pockets and either restore the Cassie state or prevent the Cassie state to collapse to the Wenzel state even at very high liquid pressures.

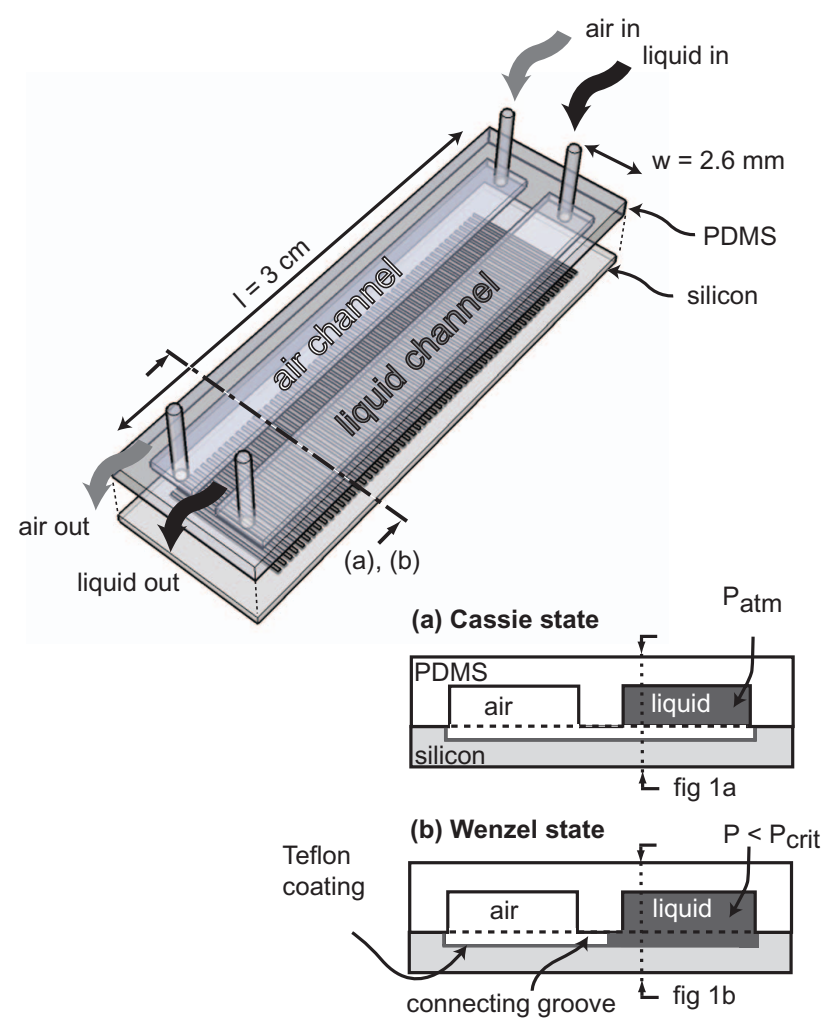

Figure 2: Schematic of the microchannel device. Parallel liquid and air channels communicate trough a perpendicular micrograting which forms a superhydrophobic texture. The device is fixed between two acrylic plates for rigidity (not shown).

\section{EXPERIMENTS AND RESULTS}

A micrograting pattern was fabricated in silicon using standard microfabrication methods. First the groove pattern was defined lithographically. Thereafter the silicon was etched using DRIE. A Teflon (1\% Teflon AF solution, DuPont) coating is applied, by spin coating and baking, to render the surface hydrophobic. The resulting static contact angle on a flat portion of the surface was measured to be $120^{\circ}$. The liquid and air channels were defined in PDMS (Sylgard 184) using soft replica moulding on a SU8 master. The PDMS channel cover was fixed to the grating with two lightly clamped acrylic plates to improve the rigidity. The top acrylic plate has access holes to the channels with steel pins fitted with polyethylene tubing $(\mathrm{ID}=0.5 \mathrm{~mm})$.

Our first, geometrically non-optimised, structure (Figure 2) consists of two parallel rectangular channels (width $w=2.6$ $\mathrm{mm}$, length $l=30 \mathrm{~mm}$, height $h=20 \mu \mathrm{m})$. Their superhydrophobic channel floors (Figure 1) consist of a micrograting positioned perpendicular to the flow direction (groove depth $d=7 \mu \mathrm{m}$, width $a=3.5 \mu \mathrm{m}$, pitch $b=8 \mu \mathrm{m}$ ). Note that the relative direction of the micrograting versus the main channel direction has not yet been optimized, and was vertical in all our tests.

Each single groove in the grating communicates with both channels. After priming one of the channels with liquid, the pressure in the trapped air pockets on the grating can be controlled by changing the air pressure in the communicating parallel air filled channel trough its air pressure ports.

Switching the air pressure allows manipulating the airwater interface and switching between the Cassie and Wenzel states, as is clearly visually observed (Figure 3). The difference in viscosity of air (Cassie state) and water (Wenzel state) in the grating results in different apparent slip lengths, and thus in altered frictional channel losses.

In the first measurement setup (Figure 4) we measured the change in mass flow due to switching between the Cassie and Wenzel states. Air pressure ports were connected to a precision syringe pump and the liquid input port to a hydrostatic pressure source $(7 \mathrm{kPa})$. The liquid mass flow trough the flow cell was measured with a high precision scale at the liquid outlet, compensating for evaporation losses.

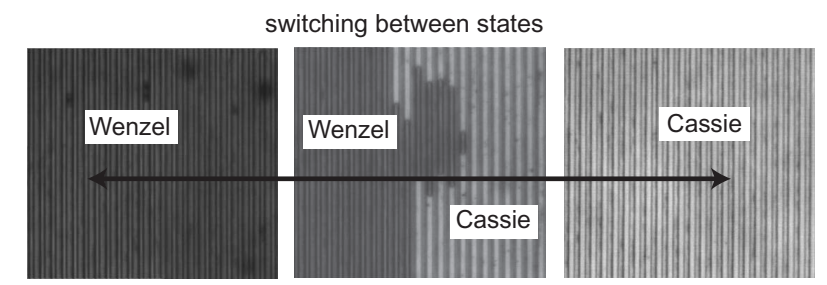

Figure 3: Photos of the grating in wet, switching and dry state.

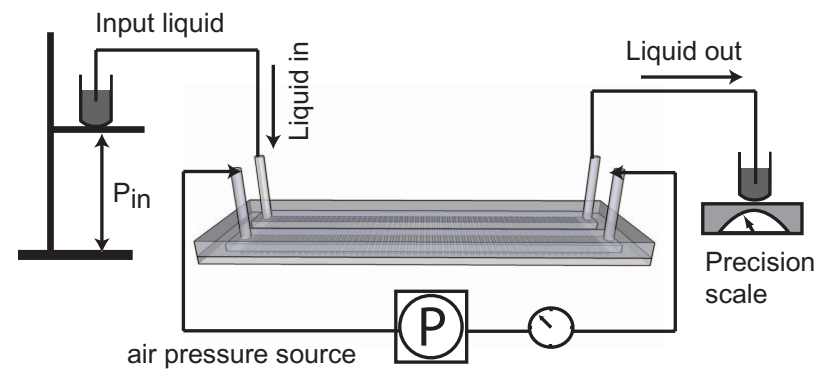

Figure 4: First experimental setup. Hydrostatic pressure drives the liquid in the liquid channel and a precision scale measures the mass flow. The lubricating air layer in the micrograting is controlled by connecting both pneumatic ports to a common pressure source. 
The state of the air pockets in the grooves could be changed by carefully increasing and decreasing the air channel pressure using the computer controlled syringe pump. The critical Laplace pressure, at which filling of the grooves occurred was measured to be $20 \mathrm{kPa}$ below the liquid pressure for the tested grating.

Applying a high positive control pressure did not result in any measurable increase in the liquid flow above the initial level. At elevated air pressures bubbles are simply introduced in the liquid channel.

When switching states, we measured a $4.8 \%$ larger flow in the Cassie state than in the Wenzel state (Figure 5), demonstrating the ability to actively manipulate the frictional losses in the channel.

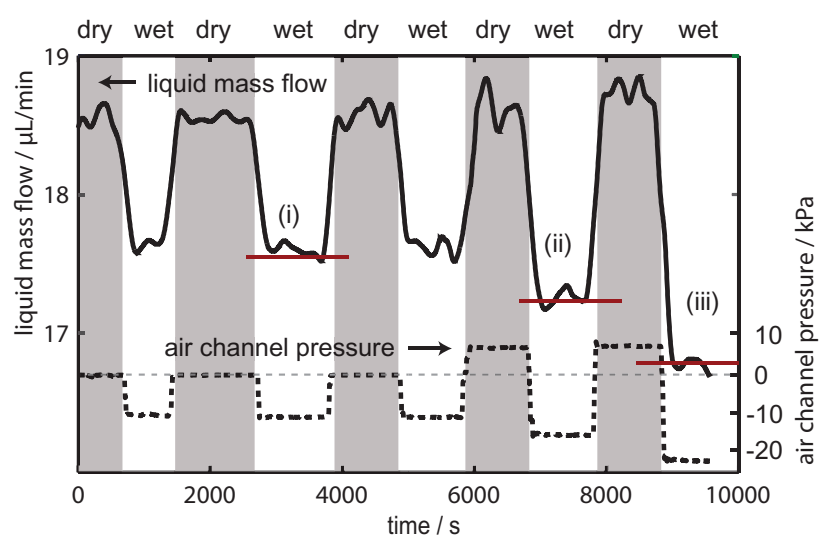

Figure 5: Results from the first experiments on a nonoptimized test structure, showing the liquid mass flow (solid) and the side channel air channel pressure (dotted). By carefully regulating the air channel pressure the curvature of the air-water interface can be repeatedly adjusted, collapsed and reconstructed. At (i) only the air pockets upstream have collapsed, at (ii) and (iii) more pockets collapse until finally all pockets have collapsed. A maximum mass flow changes accordingly with a maximum mass flow difference of $4.8 \%$ between Cassieand Wenzel state (indicated as dry and wet in figure) was measured.

The air pockets upstream started to retract and collapse first because the pressure difference over the liquid/air interface is highest at that point. This provides analogue tuning because all the air pockets will not collapse at the same time and drag reduction can be varied, as can be seen in the right part of figure 5 (i-iii), where a successively lower control pressure is applied.

In a second experiment (Figure 6) the ability of the device to withstand high liquid pressures without collapse of the air pockets was evaluated. The upstream air pressure and liquid ports were coupled to the same high pressure source and the downstream ports were open to atmospheric pressure, thus ensuring a low pressure drop over the liquid-air interface throughout the entire system.

We verified by optical inspection that the lubricating air layer (with $a=3.5 \mu \mathrm{m}$ !) remained intact at liquid pressures exceeding $60 \mathrm{kPa}$, i.e. a factor three above the previous passive Laplace pressure limit. This shows the additional ability the suggested method to create superhydrophobic surfaces with substantial drag reduction, under high liquid pressure.

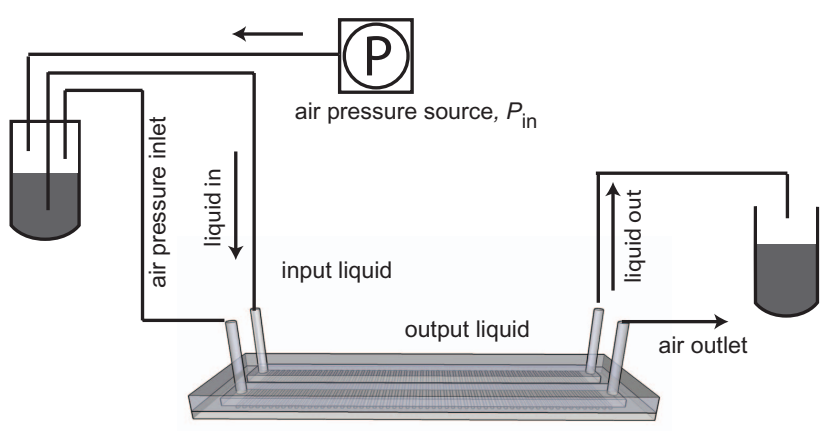

Figure 6: Second experimental setup. The upstream pneumatic ports are connected with the same pressure, as well as the downstream pneumatic and water ports. In this configuration the air-water pressure remains very low troughout the entire device and the gratings remain in the dry state, at pressures exceeding $60 \mathrm{kPa}(a=3.5 \mu \mathrm{m})$

\section{SIMULATIONS}

In an effort to model and predict the feasible effective mass flow change due to switching between Wenzel and Cassie state, a simple 2D FE-model was constructed in COMSOL Multiphysics. The model simulates a channel section with two grooves (Figure 7). Periodic boundary conditions for $u$ and $d u / d x$ were applied in the streamwise direction. A constant pressure drop over the module was set to 2.3 $\mathrm{MPa} / \mathrm{m}$ (similar to experimental conditions).

The air/liquid interface in the Cassie state was assumed to be flat and stationary with a vanishing shear stress. In the Wenzel state the liquid fill the entire grooves. The solid ridge surfaces are modeled with a no-slip boundary condition. Replacing the zero-slip condition with a small finite slip length as boundary condition, as reported in [2], did not significantly change the outcome. The properties of water and air were used.

The FE results for a geometry identical to our experimental structure (channel height $h=20 \mu \mathrm{m}$, groove width $a=3 \mu \mathrm{m}$ and pitch $b=12 \mu \mathrm{m}$ ) was a mass flow change between Cassie and Wenzel states of $4.1 \%$, in line with the experimental result of $4.8 \%$. We also showed that a FE analysis of a more optimized geometry (width $a=10 \mu \mathrm{m}$, pitch $b=12 \mu \mathrm{m}$ ) predicts over $30 \%$ flow switching capability, which demonstrates the feasibility of this method to design active microfluidic components based on active temporal and spatial manipulation of channel friction. 

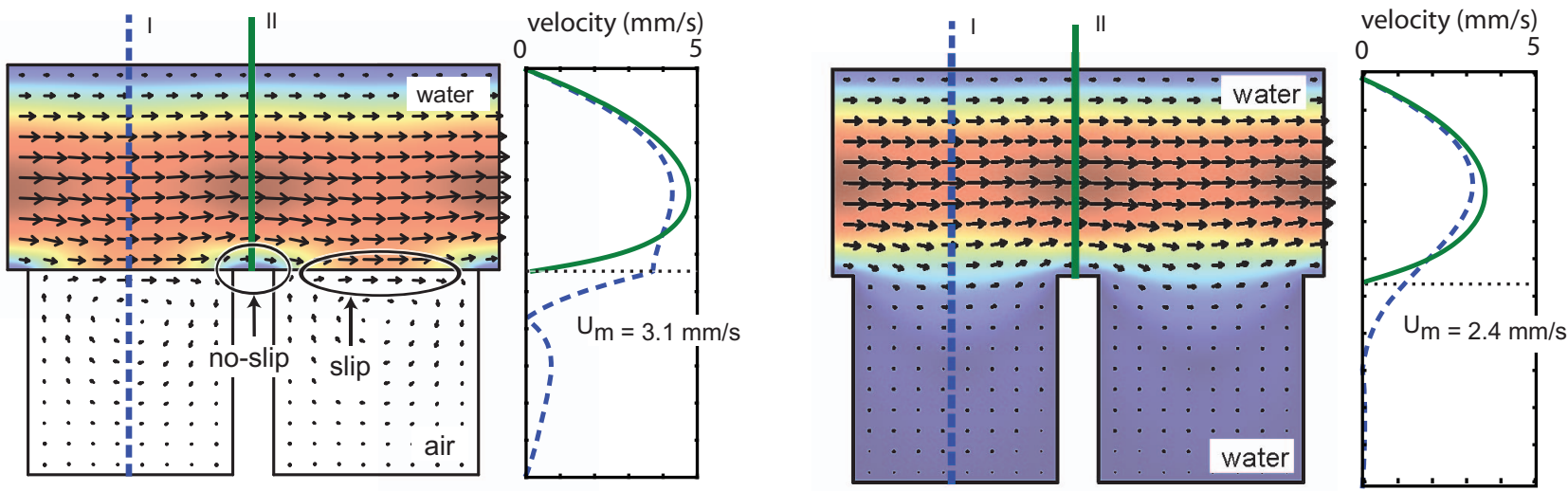

Figure 7: FE simulations of a structure with grating width $a=10 \mu \mathrm{m}$, pitch $b=12 \mu \mathrm{m}$ and channel height $h=10 \mu \mathrm{m}$ predict a theoretical mass flow switching of $30 \%$ between dry and wet state.

\section{CONCLUSION}

We successfully demonstrate active flow control in a microchannel device by pneumatic manipulation of the lubricating air pockets present at a superhydrophobic channel wall. The absolute apparent slip length can be varied with micrometer range and is independent of the liquid pressure inside the channel. Also, the system is robust, allowing active restoring of collapsed air lubrication pockets.

\section{REFERENCES}

[1] E. Lauga and H.A. Stone, J. Fluid Mech., 489 (2003), p. $55-77$

[2] J. Davies, D. Maynes, B.W. Webb, and B. Woolford, Physics of Fluids, 18 (2006)
[3] E. Schnell, J. Applied Physics, 27 (1956), pp. 11491152

[4] J. Ou, B. Perot and J.P. Rothstein, Physics of Fluids, 16 (2004), pp. 4635-4643

[5] J. Ou and J.P. Rothstein, Physics of Fluids, 17 (2005)

[6] C-H. Choi, U. Ulmanella, J. Kim, C-M. Ho and C-J. Kim, Physics of Fluids, 18 (2006) 